\title{
Transient Beauty (wabi sabi)
}

Genèse d'une créationphotographique

Transient Beauty (wabi sabi)

\section{Magali Laigne}

\section{(2) OpenEdition}

Journals

Édition électronique

URL : https://journals.openedition.org/tc/5935

DOI : $10.4000 /$ tc. 5935

ISBN : 1952-420X

ISSN : 1952-420X

Éditeur

Éditions de l'EHESS

\section{Édition imprimée}

Date de publication : 15 décembre 2011

Pagination : 256-267

ISBN : 978-2-7351-1437-5

ISSN : 0248-6016

\section{Référence électronique}

Magali Laigne, «Transient Beauty (wabi sabi) », Techniques \& Culture [En ligne], 57 | 2011, mis en ligne le 30 juin 2012, consulté le 29 septembre 2022. URL : http://journals.openedition.org/tc/5935 ; DOI : https://doi.org/10.4000/tc.5935 


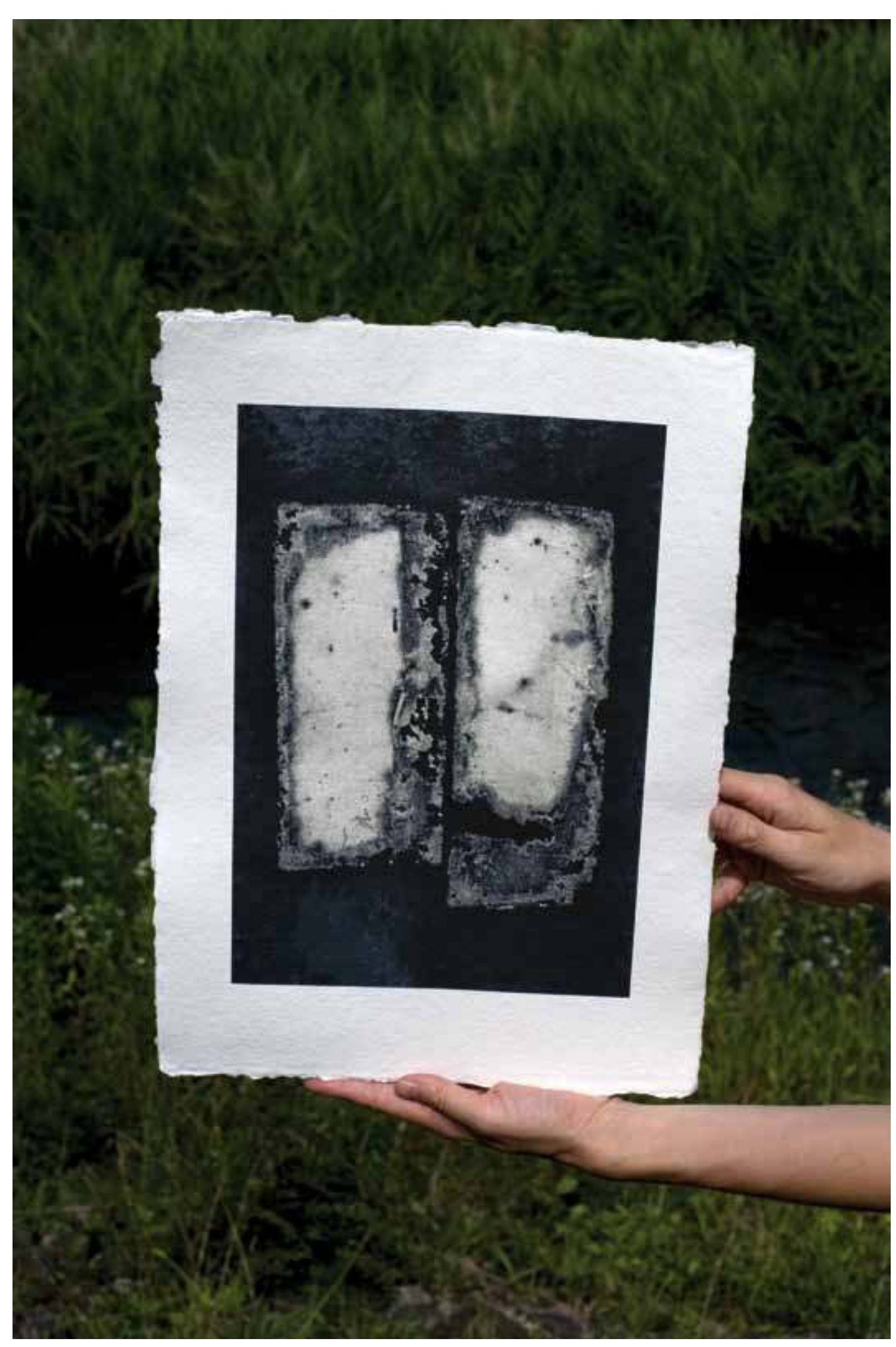

\section{TRANSIENT BEAUTY (WABI SABI)}

Genèse d'une création photographique

Lors de ma formation en graphic design à la London University of the Arts, je relis les textes de Roland Barthes et Victor Burgess, dans une analyse pratique de la relation image/texte. Je décide alors de travailler sur le banal, ce qui fait notre quotidien mais auquel nous n'accordons plus de valeur, fascinés que nous sommes par la sophistication, par les artifices de la société de consommation

M'inspirant du texte de Charles Baudelaire, « Le Joujou du pauvre », je repense alors comment un enfant, seul dans un jardin, peut recréer un monde en jouant avec des feuilles et des bouts de bois. La création est imagination. Limagination est création.

Ma démarche m'incite à utiliser non pas un appareil numérique, mais un appareil mécanique. Je réapprends le temps : temps de pose, temps de la lumière, temps de «linstant decisif », temps du developpement et temps de lattente, excitation et incertitude du cliché. Célébration de la surprise, déception ou satisfaction, acceptation de l'échec comme célébration de l'heureux accident (happy accident), impossible retour en arrière de l'instant unique.

Je suis les préceptes de Gerda $\operatorname{Taro}^{2}$ : trois prises de vue. Pas une de plus. Je suis heureuse de battre en brèche la consommation picturale du numérique avec lequel on « mitraille », on sélectionne, puis on jette, sans savourer le temps, l'instant ni même le sujet du cliché. Avec lappareil argentique chaque prise est précieuse. Il n'y en aura donc que trois.

Je croise les oeuvres d'Emmanuel Sougez ${ }^{3}$ de Ralph Gibson, mais surtout celles des femmes photographes de la Nouvelle Photographie (Ergy Landau ${ }^{4}$. Florence Henri ${ }^{5}$ Germaine Krull ${ }^{6}$, Laure Alhin Guillot ${ }^{7}$ ), de ces photographes qui ont sublime

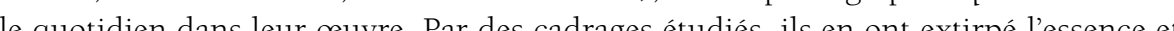
service de limagination de lhomme non pag lique et mysterieuse. La photographie au Une photographie humaniste en somme. 
Immédiatement s'impose à moi le choix d'une photographie en noir et blanc (la couleur parasite l'essence de limage, chargée de sens en elle-même) et du cadrage serré qui élimine toute contextualisation.

Mes prises de vues sont concentrées, cernées. Ce qui m'intéresse avant tout c'est le travail de cadrage qui va donner un nouveau sens à un objet, à une scène.

Parallèlement je commence aussi à étudier le développement de la narration dans une image ou dans une association d'images. Le recadrage, l'abstraction photographique suscitent l'interrogation et sollicitent l'imagination de l'audience.

La mise en relation de deux ou plusieurs photographies peut, sans aucun mot, raconter une histoire : le sujet photographique mais aussi la mise en page dans un objet, livre ou brochure. Je prends conscience de la valeur de l'espace, du blanc.

Du blanc par rapport à l'image. Du blanc qui n'est pas vide, mais qui au contraire, renforce le sens de l'image.

En novembre 2007, je participe au programme « Dialogue and commitment to the City towards creative education exchange » organise a Tokyo par le Tokyo Metropolitan Government, Tokyo Wondersite et London University of the Arts. Mes recherches prennen alors une nouvelle ampleur. J'y retrouve avec plaisir les valeurs de noir et de blanc, notamment dans le sumi- $e^{8}$ dans lequel japprends limportance de l'espace et la relation entre dit/non-dit ; explicite/implicite. Japon, civilisation du suggéré, culture du silence éloquent. Un maître de sumi-e ne représente que 60\% de son sujet (Sato 1984). Le spectateur doit rester libre de donner sens au blanc à ce qui n'apparaît pas. Importance de l'espace vierge, non pas vide, mais chargé de sens et de promesses, qui renforce ce qui est montré, mais renforce peut-être plus encore tout ce qui ne l'est pas. Me voilà bien au coeur d'une esthétique japonaise.

De même, la bichromie noir/blanc du sumi-e relève de ce même souci de libérer l'esprit et l'imagination. Comme un écho à ma démarche initiale qui considère la couleur comme parasite de l'essence du discours. La couleur contextualise, porte une charge émotive, historique, sociale que je souhaite évacuer pour ne donner à voir que l'essence même du sujet. Lartiste de sumi-e ne retouche pas, ne reprend pas son oeuvre. Il trace dans le trail d'un souffle. Il doit accepter l'imperfection. Elle lui rappelle sa place en ce monde. La perfection ne sera bien sûr jamais atteinte. Humilité. Mais dans chaque trait, porter toute sa concentration et son esprit, être pour un instant en tension extrême entre son corps et son esprit est la voie pour s'approcher de soi-même et donc des autres. Je retrouve le principe que je me suis assigné dans mon travail : choix d'accepter l'erreur, l'errance, renoncer au « tout contrôle ». Pas de retouche pas de reprise. Être libre. Libre d'accepter l'erreur. Libre de choisir son temps. Luxes que ne permet plus notre société

Accepter l'erreur, s'enthousiasmer du happy accident, célébrer l'imperfection, l'unique qui porte intrinsèquement toute sa valeur, ne plus craindre le temps dans sa dimension éphémère et fatale, ne pas revenir en arrière. Au Japon, je retrouve tous les éléments qui sous-tendent mon travail. Et ces éléments sont rassemblés dans un concept : le wabi subi. Le wabi sabi est un concept esthétique issu du zen qui unit le wabi (solitude, sim-

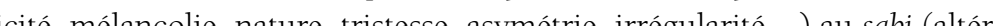

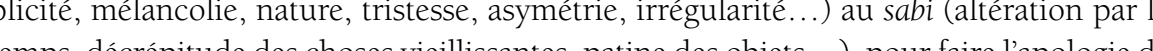

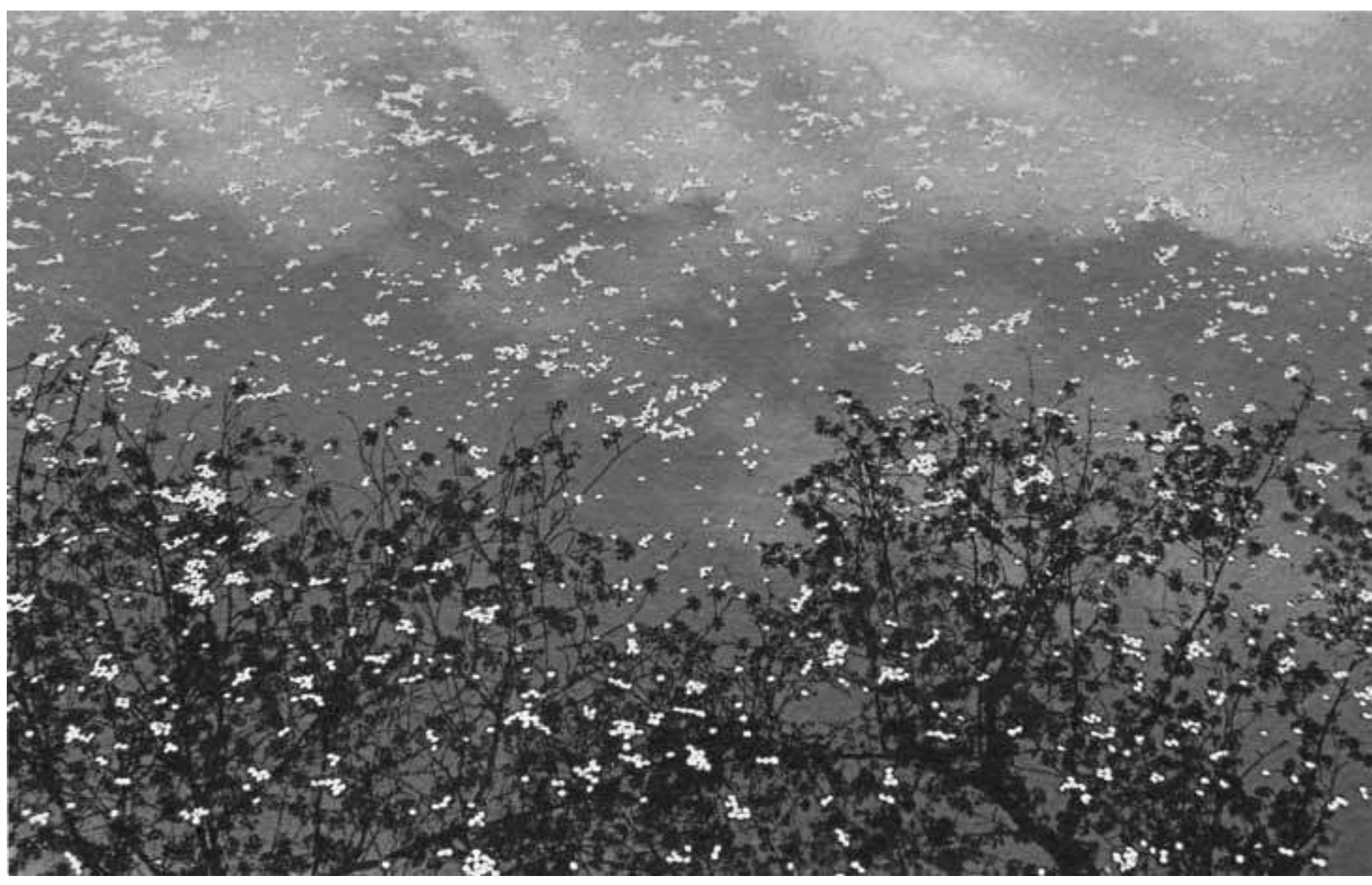


léphémère, de la fragilité, de la beauté des choses imparfaites et modestes. Ces valeurs se retrouvent dans la plupart des arts traditionnels japonais : ikebana, cérémonie du the, Pour l'exposition des photographies, je souhaitais respecter les règles du wabi sabi des éléments naturels et périssables uniquement : ni plastique, ni verre. Les photographies ne sont donc pas protégées, exposées elles-mêmes au processus de détérioration naturel des visiteurs .) simplement accrochées par des épingles à linge

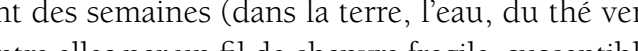
de se briser un jour, comme un fil de vie.

du wabi sabi à mon travail, j'ai tiré les photos sur un papier coton fait main, sans acide (ce qui assure une meilleure conservation des tirages), dont les irrégularités, laspect brut correspondaient à la texture que je recherchais. Par ailleurs, les impressions, en raison de lépaisseur du papier et des ses imperfections, présentent des giclures, des traces uniques a chaque tirage. Chacune des photos n'est don pas reproductible à lidentique. Chacune porte ses propres imperfections ${ }^{10}$. Chaque tirage est donc absolument et intrinséquement unique.

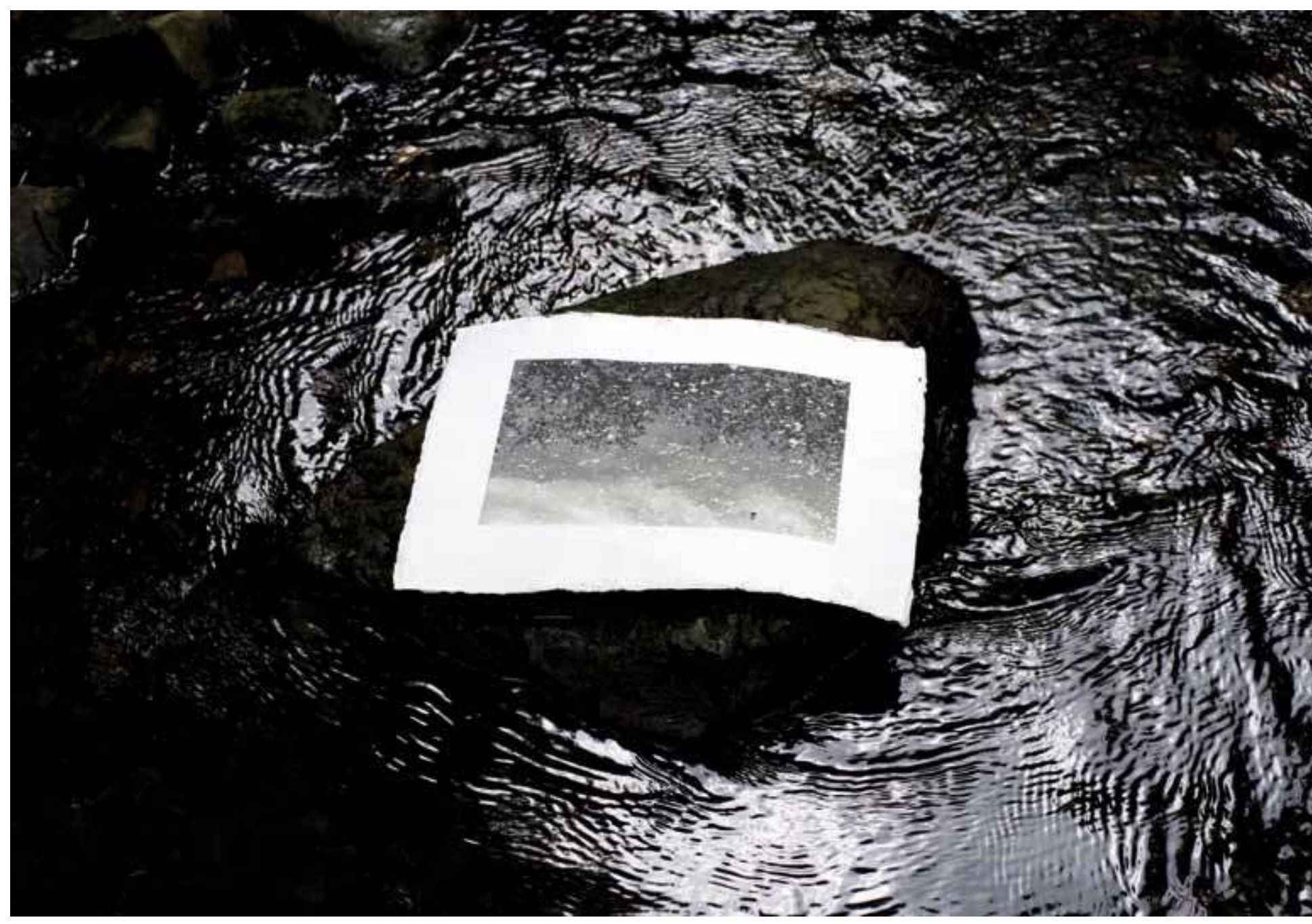



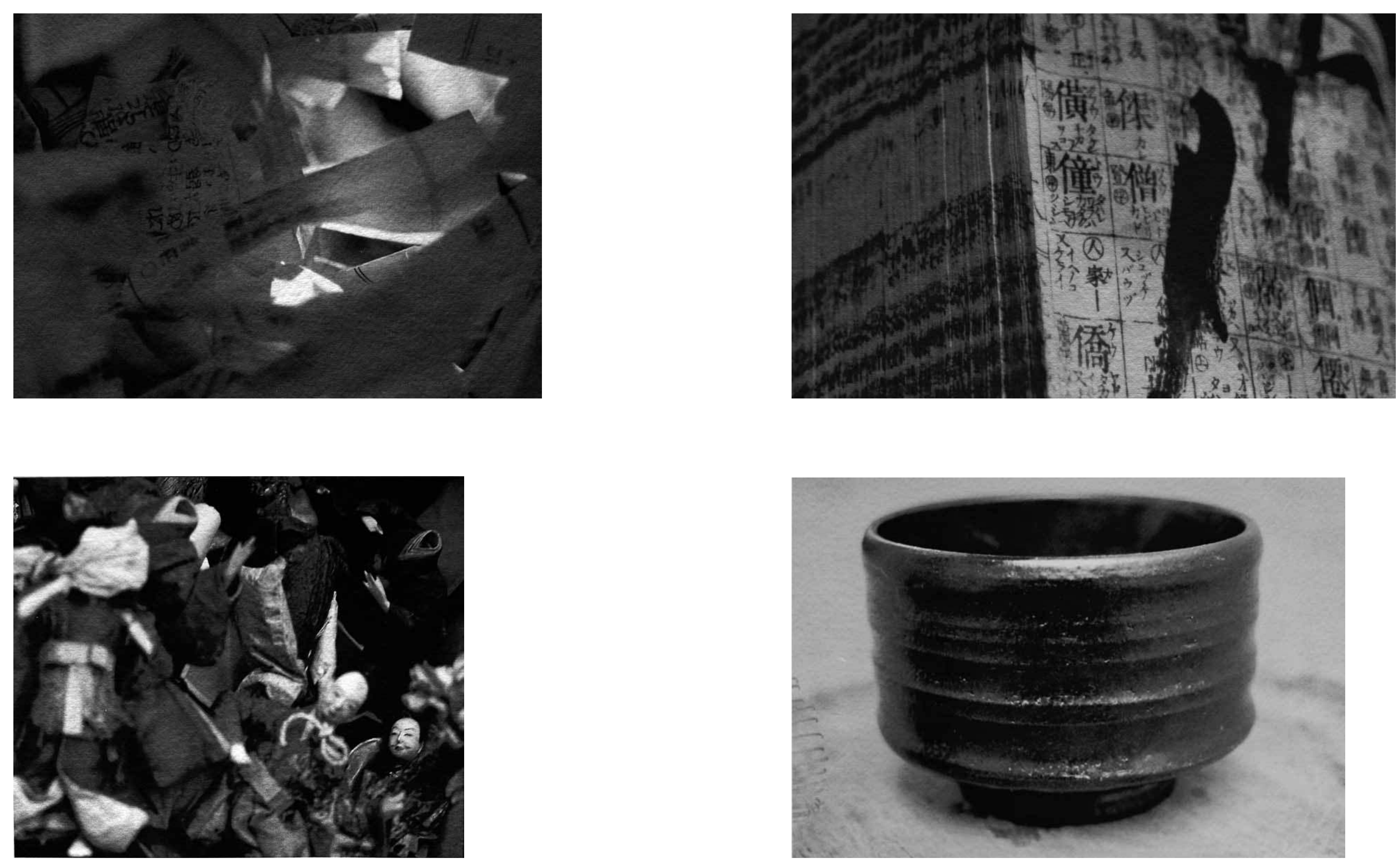


$$
\text { 口囯 }
$$


1. Okakura Kakuzo, lettré de la fin du xixe siècle, ayant étudié aux États-Unis, est un chantre de l'art et de la culture japonaise. Publié en 1906, Le livre du thé, est un traite d' introduction à la pensée et aux art

2. Gerda Taro (1910-1937) photiné aux occidene allemande, conn notamment pour ses reportages de guerre (Guerre d'Espagne). Elle fut la compagne de Robert Capa.

《... Emmanuel Sougez [qui] par la poésie dont il envebien souvent nous ne savons ni re, nous prouve que ni réfléchir "Souchez M-L.L \& Rochard A. (1993).

Ersi, « Ergy " Landau (1896-1967), photographe franco-hongroise, amie de Laszló Wholographe développe une photographie humaniste, inspiré souvent par l'architecture.

Florence Henri (1893-1982), photographe - elle étudie auprès Laszlo Moholy-Nagy - et peintre suisse. Influencée par Man Ray, Germaine Krull et And Kertèsz, elle développe un art très personnel basé sur l'utilisation de miroirs, de prismes, n'hesitant pas creer des images fragmentees qui ne sont pas sa Germaine Krull (1897

7-1985), photographe alle-
Son approche « objective » de la photographie, son attrait pour es structures métalliques, le détournement poétique et graphique des machines, le monde de l'industrie lui vaudra le surnom de "Walkyrie de fer $»$. Pendant la seconde guerre mondiale, elle dirige le service photographique de la revue France puis parcourt l'Asie d'où elle ramènera plus de 2000 phons. Aalais de Chaillot en 1967. tion au Palais de Chaillot en 1967.

, photographe Photographie et le mouvement de la Nouvelle Vision. Son activité s'étend aussi au portrail (J. Cocteau, P. Valéry, etc.) à la photographic publicitaire et de mode

8. Sumi-e, dessin à lencre aponais qui nutilise qưune seule couleur (technique du lavis).

Cetraval aboulia Londesen juin 2008 a lexposition Transient Beauty. les principes du wabi sabi son Lexposion Trusess Bdecy, dons le

. Lexpritions le cadre des japonaises, a reçu le soutien de l'ambassade du Japon. Libre a brazzaville. En 1946, elle part en Indochine.

Photos noir et blanc : M. Laigne. Photos couleurs : F. Joulian, M. Laigne.

\section{RÉFÉRENCES}

Baudelaire, C. 1975 « Le Joujou du pauvre ». In Le Spleen de Paris. Paris : Gallimard

Okakura, K. 2004 [1906] Le Livre du thé. Paris : Payot \& Rivages.

Sato, Sh. 1984 The art of Sumi-e. Appreciation, techniques et application. Tokyo, New-York \& San Franciso Kodansha International Ltd.

Souchez, M.-L. \& Rochard, A. 1993 Emmanuel Sougez, l'eminence grise. Paris : Créaphis Editions.

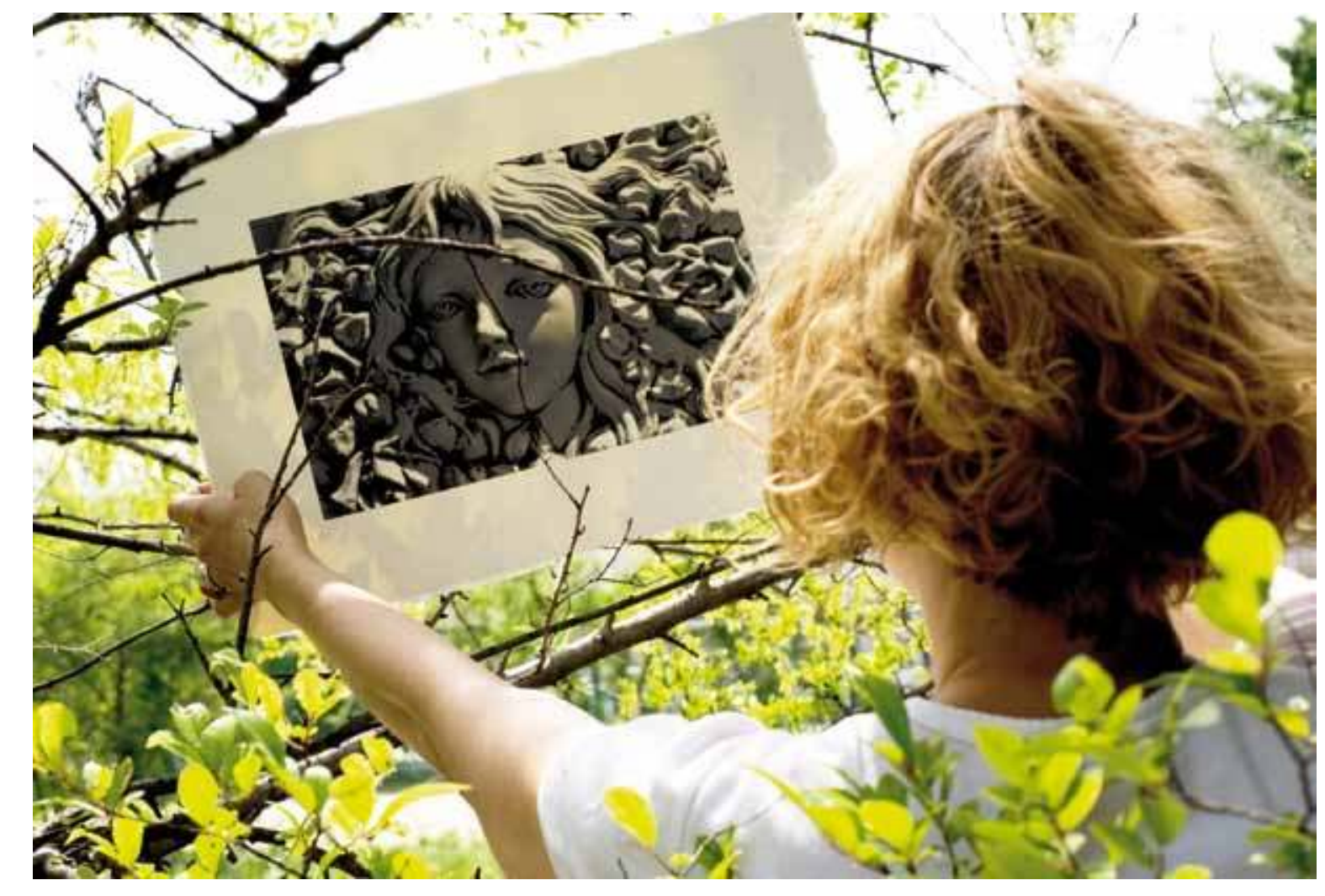

